\title{
TRANSFERÊNCIA DE RADÔNIO-222 DAS ROCHAS DO PLANALTO DE POÇOS DE CALDAS PARA AS ÁGUAS
}

\author{
DANIEL MARCOS BONOTTO \& JORGE LUÍS NEPOMUCENO DE LIMA
}

\begin{abstract}
TRANSFER OF RADON-222 FROM ROCKS OF THE POCOS DE CALDAS PLATEAU TO THE WATERS The analytical procedures developed at the Sector of Unstable Isotopes of the Laboratory of Geochemistry, which belongs to the Department of Petrology and Metallogeny located at the Institute of Geociences and Exact Sciences - Universidade Estadual Paulista "Júlio de Mesquita Filho" (UNESP) to measure the release of ${ }^{222} \mathrm{Rn}$ from rocks to the water is described. The methodology established was used to evaluate the emanation coefficient of ${ }^{222} \mathrm{Rn}$ from experiments of water-rock interaction under controlled conditions in the laboratory, which is an important parameter necessary to interpret the radioactivity related to dissolved ${ }^{222} \mathrm{Rn}$ content in waters. Four typical rocks from Poços de Caldas plateau were sampled for the experiments: phonolite, nepheline syenite, pseudoleucite tinguaite and silicified sandstone. The Ra content of these rocks was evaluated by gamma-ray spectrometry using a $\mathrm{Nal}(\mathrm{Tl})$ scintillation detector, a method that has been currently utilized in nuclear geophysics or isotope geochemistry to measure the equivalent uranium $(\mathrm{eU})$.

The Ra content in the studied rocks and the ${ }^{222} \mathrm{Rn}$ transferred to the waters due to its generation by its ${ }^{226} \mathrm{Ra}$ progenitor allowed an estimation of the emanation coefficient of ${ }^{222} \mathrm{Rn}$. A preliminary investigation on the influence

of the mass of the rock in contact .with water, of the surface area of the rock in contact with water and of the ${ }^{226} \mathrm{Ra}$ content in rocks on the amount of ${ }^{222} \mathrm{Rn}$ released to the water was also performed.
\end{abstract}

Keywords: radon, rocks, waters, emanation coefficient

RESUMO No presente trabalho são descritas as etapas analíticas desenvolvidas no Setor de Isótopos Instáveis do Laboratório de Geoquímica do Departamento de Petrologia e Metalogenia do Instituto de Geociências e Ciências Exatas da UNESP para avaliar a transferência de ${ }^{222} \mathrm{Rn}$ de rochas para a água. A metodologia implantada foi utilizada para determinar o coeficiente de emanação de ${ }^{222} \mathrm{Rn}$ a partir de experimentos de interação água-rocha sob condições controladas no laboratório, que é um parâmetro importante para interpretar a radioatividade relacionada com o teor $\mathrm{de}^{222} \mathrm{Rn}$ dissolvido nas águas.

Quatro rochas típicas do planalto de Poços de Caldas foram amostradas com o propósito de realização dos experimentos: fonólito, nefelina sienito, pseudo-leucita tinguaíto e arenito silicificado. O teor de Ra dessas rochas foi determinado por espectrometria gama com detector de cintilação de $\mathrm{Nal}(\mathrm{Tl})$, metodologia geralmente utilizada em geofísica nuclear ou geoquímica isotópicajjara a mensuração do equivalente urânio (eU).

$\mathrm{O}$ teor de Ra nas rochas estudadas e o Rn transferido para as águas devido sua geração pelo progenitor Ra permitiram estimar o coeficiente de emanação de Rn. Procedeu-se também uma investigação preliminar da influência da massa de rocha em contato com a água, da área superficial da rocha em contato com a água e do teor de $\mathrm{Ra}$ das rochas na quantidade de $\mathrm{Rn}$ transferida para a água.

Palavras-chaves: radônio, rochas, águas, coeficiente de emanação

INTRODUÇÃO A presença de gases nobres em minerais e rochas tem sido avaliada desde a descoberta da radioatividade, onde os resultados tem sido extensivamente utilizados em geociências para finalidades que variam desde a cronologia de eventos por intermédio dos isótopos de argônio até a avaliação do radônio inalado nas atividades de mineração. $\mathrm{Na}$ realidade, quando Agrícola considerou que a morte de muitos mineiros na Europa estava relacionada com o fato de que o ar pernicioso que eles respiram algumas vezes apodrece os seus pulmões (Durrance 1986), não é possível ignorar já naquela época a possibilidade da contribuição do gás Rn no episódio.

O Rn possui três isótopos de ocorrência natural, todos emissores alfa e pertencentes a séries de decaimento radioativo (Tabela 1). Eles são continuamente $\mathrm{P}^{\wedge} \mathrm{ftduzidos} \mathrm{em}$ rochas e minerais depois que seus progenitores ${ }^{223} \mathrm{Ra},{ }^{224} \mathrm{Ra}$ e ${ }^{226} \mathrm{Ra}$ emitem partículas alfa e, em decorrência de serem gases inertes, tendem a escapar dos sólidos, dirigindo-se à fase fluida circundante, como água subterrânea e/ou ar. A parcela que escapa tem sido referida como poder de emanação, fração de emanação ou coeficiente de emanação (Hahn \& Müller 1923).

A emanação de Rn de materiais geológicos tem sido investigada e revista por Wahl \& Bonner (1951) e Tanner (1964, 1980 ), porém, nos dias atuais, a auestão da radioatividade nas águas devido a presença de ${ }^{222} \mathrm{Rn}$ dissolvido assumiu proporções de grande interesse face à problemática introduzida pelos altos teores observados em águas subterrâneas provenientes de poços perfurados para abastecimento público. Apesar de que o radônio na água não constitui um risco de saúde bem documentado, sua presença contribui para a elevação do teor de $\mathrm{Rn}$ no interior de residências, o qual tem sido considerado como uma ameaça à saúde (Gesell \& Prichard 1980, Partridge et al. 1979, Bruno 1983, Prichard 1987, Nazaroff \& Nero 1988, Nazaroff et al. 1988).

Tabela 1 - Propriedades dos isótopos de Rn.

Table 1 - Properties of Rn isotopes.

\begin{tabular}{|c|c|c|c|c|c|}
\hline Isotopo & $\begin{array}{c}\text { Norine } \\
\text { Tradiclonal }\end{array}$ & Mein-vida & Strie Natural & Precurear & $\begin{array}{c}\text { Meia Vida do } \\
\text { Precursar }\end{array}$ \\
\hline${ }^{219} \mathbf{R n}$ & actinAnio & 4 segundor & $25 \sqrt{U}$ & ${ }^{23} 3^{3} \mathrm{Fan}_{\mathrm{a}}$ & 11,43 dias \\
\hline${ }^{220} \mathrm{Rn}$ & torofaio & 55 segundos & ${ }^{232} \mathrm{Th}$ & ${ }^{22} \mathrm{Ra}$ & 3,64 dias \\
\hline${ }^{2 m} \mathrm{Rn}$ & radönio & 3,82 dias & ${ }^{2} \mathbb{B}_{U}$ & ${ }^{2 x} \mathrm{Ra}$ & 1602 anos \\
\hline
\end{tabular}

Dessa forma, o conhecimento básico envolvendo a transferência de ${ }^{222} \mathrm{Rn}$ de minerais e rochas para a água, visando melhor compreender os mecanismos de sua mobilização, constitui um tema de extrema relevância atual, inserindo-se nesse contexto o presente trabalho. O objetivo da investigação consiste em conduzir experimentos sob condições controladas em laboratório, visando determinar o coeficiente de emanação de Rn da fase sólida para a líquida e avaliar os principais parâmetros relacionados com a sua transferência, em virtude 
dos processos de interação água-rocha. Ressalta-se que poucos estudos foram realizados no Brasil até o momento com este propósito, contribuindo o trabalho para a geração de dados que propiciem interpretações mais apropriadas da radioatividade em águas subterrâneas devido ao ${ }^{222} \mathrm{Rn}$ dissolvido.

A área selecionada para amostragem constituiu o maciço alcalino de Poços de Caldas (Fig. l) conhecido por apresentar diferentes tipos de mineralizações radioativas, a qual também se prestou à coleta de amostras para a realização de ensaios de lixiviação objetivando caracterizar a composição química de soluções representativas de águas de diferentes aqüíferos (Cruz \& Peixoto 1989).

AMOSTRAGEM O maciço alcalino de Poços de Caldas apresenta uma forma circular com um diâmetro médio de 33



Figura 1 - Localização das cidades de Poços de Caldas e Águas da Prata no planalto de Poços de Caldas (MG), segundo Holmes et al. (1991).

Figure 1 - Location of Poços de Caldas and Águas da Prata cities within the Poços de Caldas plateau, according to Holmes et al. (1991). 
$\mathrm{Km}$, área de aproximadamente $800 \mathrm{Km}^{2}$, altitude entre 1300 e $1600 \mathrm{~m}$ e topografia caracterizada por vales, montanhas e colinas revestidas por gramíneas. $\mathrm{O}$ planalto é uma estrutura anelar de idade mesozóica abrangendo uma suite de rochas alcalinas vulcânicas e plutônicas, principalmente fonólitos e nefelina sienitos. A história evolutiva começa com um vulcanismo principal envolvendo ankaratritos, lavas fonolíticas e rochas vulcanoclásticas, seguido por uma subsidência da caldeira e intrusão de nefelina sienitos formando diques anelares menores e estruturas circulares, e, finalmente, a intrusão de nefelina sienitos portadores de eudialita (Ellert 1959). A implantação deste complexo alcalino se iniciou no Cretáceo Superior (87 Ma) e foi evoluindo em fases sucessivas até $60 \mathrm{Ma}$ (Bushee 1971).

Diferentes tipos de mineralizações radioativas foram identificadas (Oliveira 1974) em vários setores do planalto, por exemplo: ocorrência de tório e terras raras do Morro do Ferro, quase no centro da área; mineralização zirconífera do Serrote e mineralização molibdo-uranífera do Campo do Agostinho e Campo do Cercado C-09 (Mina Osamu Utsumi) nos limites sul, sudoeste e oeste da área; mineralização molibdo-uranífera em coexistência com filões de caldasito uranífero do Campo de Taquari, nos limites sudeste e leste da área.

Para a realização dos experimentos, selecionaram-se amostras não intemperizadas de rochas ígneas, importantes tanto pelo fato de representarem litotipos de maior ocorrência no planalto, quanto por estarem relacionados com o possível caminho de circulação das águas subterrâneas até sua surgência como fontes. Tais litotipos são: fonólito (extrusiva, procedente da Cascata das Antas no município de Poços de Caldas), nefelina sienito (plutônica, procedente da mesma localidade) e pseudo-leucita tinguaíto (hipoabissal, procedente de pedreira próxima à Represa Bortolan no município de Poços de Caldas)

Além disso, conduziu-se o estudo em amostra de arenito silicificado também não intemperizada procedente da Fonte Villela no município de Águas da Prata, localizado no Estado de São Paulo e borda ocidental do planalto de Poços de Caldas (Fig. 1). A Fonte Villela emerge ao pé de um paredão nesta litologia, através de fraturas no sentido vertical e horizontal. A escolha deveu-se ao fato de que as águas dessa fonte são muito conhecidas devido a elevada presença de $\mathrm{Rn}-222$ dissolvido.

ÁREA SUPERFICIAL ESPECÍFICA DAS AMOSTRAS Para se avaliar a liberação do Rn-222 pelas amostras de rochas descritas, cada uma delas foi inicialmente triturada até uma granulometria entre 4 e $8 \mathrm{~mm}$. Os fragmentos obtidos foram separados e submetidos a uma análise de distribuição do tamanho para se estimar a área superficial de contacto entre a água e a rocha nos experimentos conduzidos.

A distribuição de massa dos fragmentos foi efetuada a partir de uma fração do total de material triturado, consistindo na pesagem individual de 100 partículas para cada uma das quatro rochas analisadas. Na Tabela 2 constam as classes de massa dos fragmentos, as quais estão dispostas em progressão geométrica. A massa média no intervalo de classe considerado e a freqüência de ocorrência também estão representados.

A área superficial da distribuição de fragmentos foi estimada com base na metodologia empregada por Bonotto \& Andrews (1993). Supôs-se inicialmente uma geometria esférica média, para a qual a área superficial específica, $\mathbf{S}$, é dada pela equação:

$$
S=\frac{4 \pi r^{2}}{\frac{4}{3} \pi r^{3} \rho}=\frac{3}{\rho r}
$$

onde: $\mathrm{p}$ é a densidade da rocha $\left(\mathrm{g} / \mathrm{cm}^{3}\right), \mathrm{r}$ é o raio do fragmento $(\mathrm{cm})$ e $\mathrm{S}$ é dado em $\mathrm{cm}^{2} \mathrm{~g}^{-1}$. A densidade de cada amostra foi determinada no laboratório, tendo sido obtidos os valores de $2,73 \mathrm{~g} / \mathrm{cm}^{3}, 3,03 \mathrm{~g} / \mathrm{cm}^{3}, 2,65 \mathrm{~g} / \mathrm{cm}^{3}$ e $2,69 \mathrm{~g} / \mathrm{cm}^{3}$, respectivamente, para o arenito silicificado, nefelina sienito, fonólito e pseudo-leucita tinguaíto.

Para um fragmento de massa $\mathbf{m}$, o raio pode ser calculado a partir da expressão:

$$
r=\left(\frac{3 m}{4 \pi \rho}\right)^{1 / 3}
$$

A área superficial específica é obtida pela substituição dessa equação em (1), isto é:

$$
\mathbf{S}=\left(\frac{3}{\rho}\right) \cdot\left(\frac{4 \pi \rho}{3 m}\right)^{1 / 3}=\left(\frac{36 \pi}{\rho^{2} m}\right)^{1 / 3}
$$

A área superficial específica de cada amostra correspondeu à soma da área superficial do grupo inserida na última coluna da Tabela 2 dividida pela massa dos 100 fragmentos utilizados para a confecção da distribuição de classes de massa. Os valores obtidos estão inseridos na Tabela 3.

INTERAÇÃO ÁGUA-ROCHA Para a realização dos experimentos de transferência de $\mathrm{Rn}-222$ das rochas para a água, os fragmentos triturados foram inseridos em frascos de 1 litro preenchidos com água destilada e vedados com rolha de borracha contendo dois tubos de nylon de $5 \mathrm{~mm}$ de diâmetro acoplados a mangueira de neoprene. Esses tubos são usados para se fazer a conexão com o sistema de degaseificação, sendo um deles vedado numa extremidade e perfurado com orifícios de $1 \mathrm{~mm}$ de diâmetro. As mangueiras de saída foram fechadas com pinças de Hoffmann e nos frascos ainda foram afixados rótulos contendo a identificação da amostra e a data em que foram vedados. Na Tabela 4 consta a massa total dos fragmentos utilizados em cada experimento e a área superficial total de contato entre a rocha e a água.

Conforme Andrews \& Wood (1972), a dissolução de Rn222 pelas águas ocorre predominantemente na interface águarocha por um processo de recuo. A atividade do $\mathrm{Rn}-222 \mathrm{em}$ solução aumenta com o tempo até que seja atingido um valor de equilíbrio determinado pela taxa de decaimento de seu gerador na superfície da rocha, isto é, o Ra-226. Esse equilíbrio é alcançado quando o tempo de residência da água em contato com a rocha excede 25 dias, de acordo com a equação:

$$
\mathbf{A}_{\mathbf{t}}=\mathbf{A}_{\mathrm{e}}\left(1-\mathbf{e}^{\lambda t}\right)
$$

onde $A_{t}$ e $A_{e}$ são, respectivamente, a atividade de $\mathrm{Rn}-222$ num instante $\mathbf{t}$ e no equilíbrio e $\lambda$, é a constante de decaimento do $\mathrm{Rn}-222$.

Por este motivo, durante a condução dos experimentos em laboratório, os frascos contendo os fragmentos de rocha e água destilada permaneceram armazenados por um período mínimo de 26 dias. Decorrido o período de tempo necessário, os frascos foram inseridos na linha de extração de radônio, com o propósito de se recuperar o gás Rn-222 presente na água e proveniente da rocha. Este gás foi transferido para câmara de cintilação, conforme metodologia descrita por Lima \& Bonotto (1995), e então para o sistema de detecção a fim de que fosse mensurada a sua atividade na água. Na Tabela 5 constam os resultados obtidos onde $\mathbf{t}$ corresponde ao intervalo de tempo entre o início do processo de extração e o final da realização da medida. 
Tabela 2 - Classes de massa dos fragmentos das rochas utilizadas nos experimentos de transferência de 222 Rn para a água Table 2 - Mass distribution of gravels utilized in the experiments of $\mathrm{Rn}$ transfer to the water.

\begin{tabular}{|c|c|c|c|c|c|c|}
\hline Amostra & $\begin{array}{c}\text { Classe de massa } \\
\text { do fragmento } \\
\text { (g) }\end{array}$ & \begin{tabular}{|c|} 
Massa média \\
(g)
\end{tabular} & Frequência & $\begin{array}{c}\text { Raio do } \\
\text { fragmento } \\
\text { (cm) }\end{array}$ & $\begin{array}{c}\text { Area do } \\
\text { fragmento } \\
\left(\mathrm{cm}^{2}\right)\end{array}$ & $\begin{array}{c}\text { Área superficial } \\
\text { do grupo } \\
\left(\mathrm{cm}^{2}\right)\end{array}$ \\
\hline Arenito & $0,025-0,043$ & 0,034 & 1 & 0,144 & 0,260 & 0,260 \\
\hline \multirow[t]{6}{*}{ silicificado } & $0,043-0,074$ & 0,075 & 1 & 0,187 & 0,439 & 0,439 \\
\hline & $0,074-0,127$ & 0,100 & 18 & 0,206 & 0,533 & 9,594 \\
\hline & $0,127-0,219$ & 0,173 & 27 & 0,247 & 0,767 & 20,709 \\
\hline & $0,219-0,377$ & 0,298 & 31 & 0,296 & 1,101 & 34,131 \\
\hline & $0,377-0,650$ & 0,513 & 18 & 0,355 & 1,584 & 28,512 \\
\hline & $0,650-1,121$ & 0,885 & 4 & 0,426 & 2,280 & 9,120 \\
\hline Nefelina & $0,080-0,113$ & 0,096 & 8 & 0,196 & 0,046 & 0,368 \\
\hline \multirow[t]{6}{*}{ sienito } & $0,113-0,160$ & 0,136 & 19 & 0,220 & 0,608 & 11,552 \\
\hline & $0,160-0,226$ & 0,193 & 27 & 0,248 & 0,773 & 20,871 \\
\hline & $0,226-0,320$ & 0,273 & 27 & 0,278 & 0,971 & 26,217 \\
\hline & $0,320-0,453$ & 0,386 & 13 & 0,312 & 1,223 & 15,899 \\
\hline & $0,453-0,641$ & 0,547 & 4 & 0,351 & 1,548 & 6,192 \\
\hline & $0,641-0,908$ & 0,774 & 1 & 0,394 & 1,951 & 1,951 \\
\hline \multirow[t]{7}{*}{ Fonólito } & $0,030-0,048$ & 0,039 & 1 & 0,152 & 0,290 & 0,290 \\
\hline & $0,048-0,077$ & 0,062 & 4 & 0,177 & 0,394 & 0,708 \\
\hline & $0,077-0,124$ & 0,100 & 18 & 0,208 & 0,544 & 9,792 \\
\hline & $0,124-0,199$ & 0,161 & 36 & 0,244 & 0,748 & 26,928 \\
\hline & $0,199-0,320$ & 0,259 & 28 & 0,286 & 1,028 & 28,784 \\
\hline & $0,320-0,514$ & 0,417 & 12 & 0,335 & 1,410 & 16,920 \\
\hline & $0,514-0,826$ & 0,670 & 1 & 0,392 & 1,931 & 1,931 \\
\hline Pseudo- & $0,070-0,103$ & 0,086 & 1 & 0,197 & 0,488 & 0,488 \\
\hline leucita & $0,103-0,152$ & 0,127 & 4 & 0,224 & 0,630 & 2,520 \\
\hline \multirow[t]{5}{*}{ tinguaito } & $0,152-0,225$ & 0,188 & 23 & 0,225 & 0,817 & 18,791 \\
\hline & $0,225-0,332$ & 0,278 & 23 & 0,291 & 1,064 & 24,472 \\
\hline & $0,332-0,491$ & 0,411 & 28 & 0,332 & 1,385 & 38,780 \\
\hline & $0,491-0,725$ & 0,608 & 18 & 0,378 & 1,795 & 32,310 \\
\hline & $0,725-1,071$ & 0,898 & 3 & 0,430 & 2,323 & 6,969 \\
\hline
\end{tabular}

ESPECTROMETRIA GAMA DAS AMOSTRAS Para a determinação do coeficiente de emanação de ${ }^{222} \mathrm{Rn}$ da fase sólida para a líquida também é necessário conhecer o teor de ${ }^{226} \mathrm{Ra}$ nas rochas, o que pode ser aproximadamente efetuado a partir da espectrometria gama, que é uma técnica empregada para a medida do equivalente urânio (eU), a qual é de uso corrente em geofísica nuclear ou geoquímica isotópica e tem sido utilizada por vários investigadores no Brasil (Pereira 1977, Hiodo 1989, Pascholati 1990, Ferreira 1991).

Características gerais do método Os nuclídeos pertencentes à série do urânio emitem radiações gama com energias características. Nesta série, equilíbrios radioativos a partir do Ra-226 são atingidos em intervalos de tempo curtos do ponto de vista geológico, desde que não haja escape do gás $\mathrm{Rn}-222$. Na condição do sistema estar fechado, a atividade do
Bi-214 presente deve ser igual à atividade do Ra-226 na amostra.

As determinações quantitativas das concentrações equivalentes de urânio $(\mathrm{eU})$ nas rochas tem sido feitas utilizando-se a emissão gama natural do Bi-214 com energia da ordem de $1,76 \mathrm{MeV}$, a qual é muito apropriada para estas análises.

Na figura 2 é apresentado um espectro obtido por um espectrômetro gama empregando um cristal de $\mathrm{Nal}(\mathrm{Tl})$ de $3 "$ x 3 " a partir de uma amostra de pechblenda em equilíbrio radioativo secular. A área assinalada na figura refere-se à região espectral (ou janela) cujo intervalo de energia varia de 1,66 a $1,86 \mathrm{MeV}$ e corresponde ao radionuclídeo Bi-214, a qual é normalmente usada para a quantificação do urânio e será utilizada neste trabalho para a medida do teor de Ra-226.

O sistema empregado baseia-se na detecção, amplificação e contagem dos fótons gerados pela interação da radiação 
Tabela 3 - Area superficial específica das rochas utilizadas nos experimentos de transferência de ${ }^{222} \mathrm{Rn}$ para a água. Table 3 - Specific surface area of the rocks utilized in the experiments of ${ }^{222} \mathrm{Rn}$ transfer to the water.

\begin{tabular}{|l|c|c|c|}
\hline \multicolumn{1}{|c|}{ Amostra } & $\begin{array}{c}\text { Massa dos } \\
\text { fragmentos } \\
(\mathbf{g})\end{array}$ & $\begin{array}{c}\text { Área } \\
\text { superficial do } \\
\text { grupo }\left(\mathbf{c m}^{\mathbf{2}}\right)\end{array}$ & $\begin{array}{c}\text { Área superficial } \\
\text { específica } \\
\left(\mathbf{c m}^{\mathbf{2}} \mathbf{g}^{-\mathbf{1}}\right)\end{array}$ \\
\hline Arenito silicificado & 27,34 & 102,76 & 3,76 \\
\hline Nefelina sienito & 24,24 & 83,05 & 3,43 \\
\hline Fonólito & 21,17 & 85,35 & 4,03 \\
\hline Pseudo-leucita tinguaíto & 34,93 & 124,35 & 3,56 \\
\hline
\end{tabular}

Tabela 4 - Massa total e área superficial total das amostras utilizadas nos experimentos de transferência de ${ }^{222} \mathrm{Rn}$ para a água.

Table 4 - Total mass and total surface area of the samples utilized in the experiments of ${ }^{222} \mathrm{Rn}$ transfer to the water.

\begin{tabular}{|l|c|c|}
\hline \multicolumn{1}{|c|}{ Amostra } & $\begin{array}{c}\text { Massa total } \\
(\mathrm{g})\end{array}$ & $\begin{array}{c}\text { Área superficial total } \\
\left(\mathrm{cm}^{2}\right)\end{array}$ \\
\hline Arenito silicificado & 627 & 2357,52 \\
Fonólito & 562 & 2264,86 \\
Nefelina sienito & 695 & 2383,85 \\
Pseudo leucita tinguaíto & 663 & 2360,28 \\
\hline
\end{tabular}

gama com um cristal de $\mathrm{Nal}(\mathrm{Tl})$ de $50 \mathrm{~mm}$ de diâmetro por $5 \mathrm{Q} \mathrm{mm}$ de espessura, acoplado a uma válvula fotomultiplicadora. Este conjunto encontra-se diretamente unido a um pré-amplificador de fabricação Ortec, o qual foi inserido numa blindagem de chumbo para redução das contagens de fundo. A polarização da válvula fotomultiplicadora foi efetuada com a fonte de alta tensão operando a +950 volts. Para amplificação, utilizou-se unidade da Ortec que possui saída linear de pulsos cujo formato foi selecionado para unipolar; sua impedância de saída é de cerca de $0,5 \mathrm{ohm}$. Esse amplificador foi acoplado a um analisador multicanal de 1024 canais, o qual registrou o número de contagens realizadas por canal; a integral dos picos de interesse foi efetuada diretamente no display do multicanal, estando representado no diagrama da Figura 3 as unidades constituintes desse sistema. Os resultados oriundos da integração dos picos foram empregados para a determinação do teor de Ra-226 a partir da curva de calibração ilustrada Figura 5.
Preparação das amostras, padrões e curvas de calibração A preparação das amostras envolveu a sua trituração em moinho de rolo e peneiramento até granulação da ordem de $0,5 \mathrm{~mm}$. Após pesagem, elas foram acondicionadas em caixas cilíndricas com $62 \mathrm{~mm}$ de diâmetro por $23 \mathrm{~mm}$ de altura, que, por sua vez, foram seladas para evitar escape do gás radônio. Nesta condição elas permaneceram durante um período de 26 dias, tempo necessário para o Ra-226 atingir o equilíbrio radioativo com o $\mathrm{Rn}-222$ e este com os seus filhos (Bi-214 inclusive).

Para a obtenção dos teores de Ra-226 é necessário o uso de padrões com concentração conhecida desse nuclídeo. Com esse propósito, três padrões fornecidos pelo NBL (New Brunswick Laboratory) do Departamento de Energia dos Estados Unidos foram também acondicionados em recipientes selados idênticos aos descritos no item anterior e mantidos armazenados durante o mesmo intervalo de tempo. Tais padrões, designados de 106-A, 107-A e 109-A, possuem massa de $50 \mathrm{~g}$ e são constituídos de mistura de areia monazítica e silica; os valores de suas concentrações de Ra-226 são, respectivamente, $0,141 \mathrm{ppb}, 0,0140 \mathrm{ppb}$ e $0,00144 \mathrm{ppb}$.

O sistema de espectrometria gama utilizado foi calibrado em energia por intermédio das seguintes fontes radioativas disponíveis no laboratório: Cs-137 e Co-60. Além delas, empregou-se cloreto de potássio $(\mathrm{KC} 1)$ inserido em caixa cilíndrica similar à descrita, o qual é portador do radionuclídeo $\mathrm{K}-40$. A meia-vida desse nuclídeo é de $1,31 \times 10$ anos e sua abundância em relação ao potássio total é de 0,0119\% (Dalrymple \& Lanphere 1969).

Tanto o K-40 quanto o Cs-137 ou Co-60 emitem radiações gama de energias apropriadas para a calibração do sistema, isto é, podem ser extrapoladas facilmente para a região espectral de interesse correspondente à do radionuclídeo $\mathrm{Bi}-214$. Na Figura 4 está representada a curva de calibração de energia em função do canal traçada a partir dos dados obtidos, onde também está indicado o canal esperado para o pico do Bi-214.

Os padrões acondicionados conforme descrito anteriormente foram introduzidos no sistema espectrométrico ilustrado Figura 3 para a obtenção dos dados referentes às contagens no pico do Bi-214 de $1,76 \mathrm{MeV}$ de energia. As contagens líquidas determinadas subtraindo as contagens totais no pico das contagens do fundo foram, então, convertidas para intensidade efetiva, isto é, contagens massa" (em gramas) tempo" (em minutos), constando na Figura 5 a curva de calibração da concentração de Ra-226 em função da intensidade efetiva, onde tanto na ordenada quanto na abcissa os

Tabela 5 - Atividade do ${ }^{222}$ Rn liberado para a água a partir das amostras de rochas. Todas as incertezas analiticas correspondem a um desvio padrão de $1 \sigma$.

Table 5 - Activity of ${ }^{222} \mathrm{Rn}$ released to the water from the rock samples. All reported analytical uncertainties correspond to la standard deviation.

Table 5 - Activy of ${ }^{222} \mathrm{Rn}$ release to the water from the rock samples. All reported analytical uncertainties correspon to $1 \sigma$ standard deviation.

\begin{tabular}{|c|c|c|c|c|c|}
\hline Amostra & $\begin{array}{c}\text { Taxa de } \\
\text { contagem total } \\
(\mathbf{c p m}) \\
\end{array}$ & $\begin{array}{c}\text { Taxa de contagem } \\
\text { líquida } \\
\text { (cpm) }\end{array}$ & $\begin{array}{c}t \\
(\min )\end{array}$ & $\begin{array}{c}\text { Taxa de contagem } \\
\text { corrigida } \\
(\mathrm{cpm}) \\
\end{array}$ & $\underset{*}{\text { Atividade (pCi) }}$ \\
\hline \begin{tabular}{|l|} 
Arenito \\
silicificado
\end{tabular} & $\begin{array}{r}1699,00 \\
\pm 41,22 \\
\end{array}$ & $\begin{array}{r}1697,44 \\
\pm 41,24 \\
\end{array}$ & 184 & $\begin{array}{r}1737,20 \\
\pm 42,21 \\
\end{array}$ & $\begin{array}{r}4116,59 \\
\pm 99,98 \\
\end{array}$ \\
\hline Nefelina sienito & $\begin{array}{l}387,10 \\
\pm 1,97 \\
\end{array}$ & $\begin{array}{l}381,86 \\
\pm 2,08 \\
\end{array}$ & 254 & $\begin{array}{l}394,26 \\
\pm 2,15 \\
\end{array}$ & $\begin{array}{r}1752,28 \\
\pm 40,09 \\
\end{array}$ \\
\hline Fonólito & $\begin{array}{r}653,30 \\
\pm 2,55 \\
\end{array}$ & $\begin{array}{r}651,74 \\
\pm 2,58 \\
\end{array}$ & 1097 & $\begin{array}{r}748,22 \\
\pm 2,96 \\
\end{array}$ & $\begin{array}{l}1773,03 \\
\pm 22,15 \\
\end{array}$ \\
\hline $\begin{array}{l}\text { Pseudo-leucita } \\
\text { tinguaíto }\end{array}$ & $\begin{array}{r}194,30 \\
\pm 1,39 \\
\end{array}$ & $\begin{array}{l}191,76 \\
\pm 1,45 \\
\end{array}$ & 1147 & $\begin{array}{r}221,54 \\
\pm 1,67 \\
\end{array}$ & $\begin{array}{r}848,80 \\
\pm 17,47 \\
\end{array}$ \\
\hline
\end{tabular}

*pCi $=$ picocurie $(1 \mathrm{pCi}=0,037$ desintegrações por segundo). 


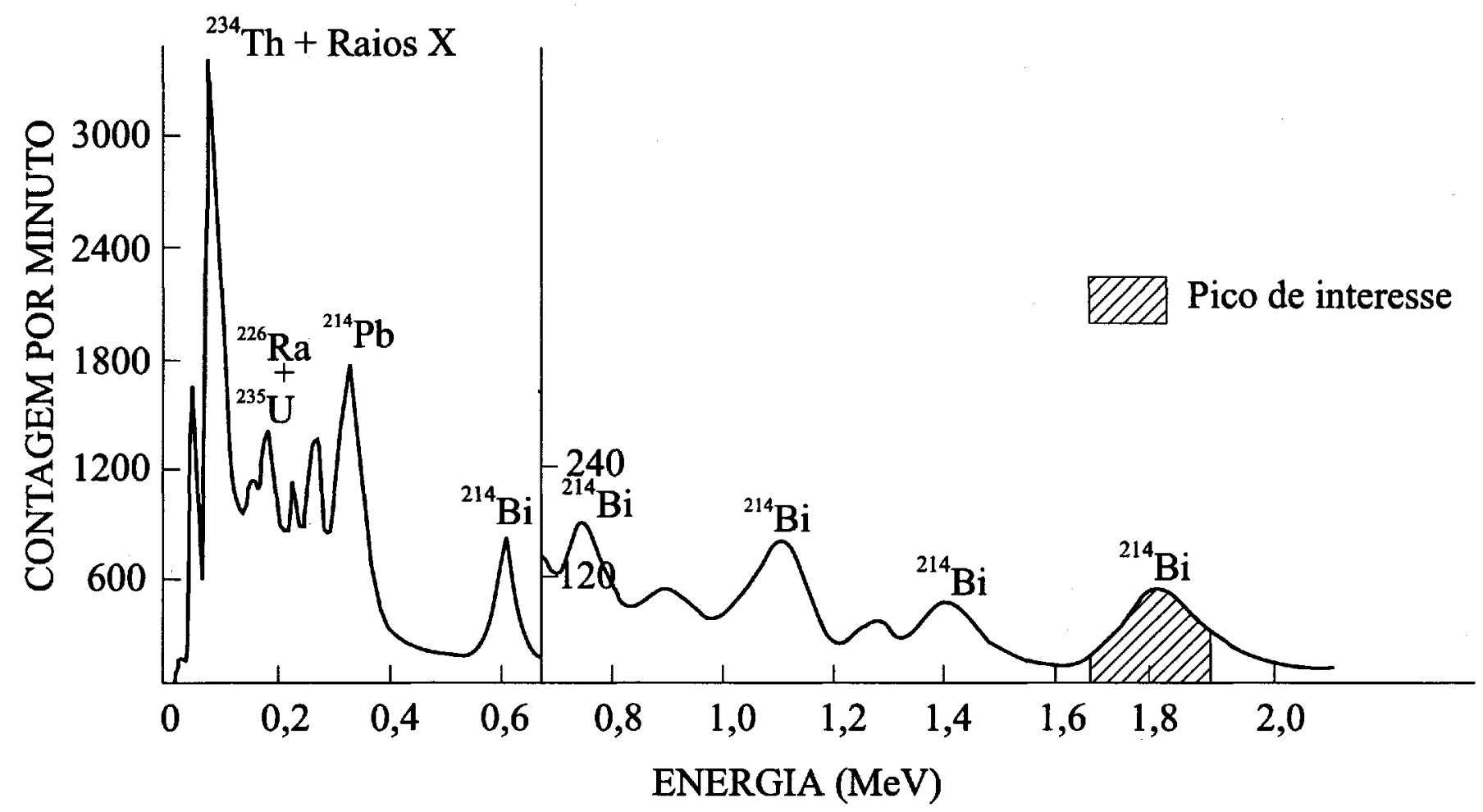

Figura 2 - Espectro gr ama gama da série natural do ${ }^{238}$ U em equilibrio radioativo obtido a partir de detector de Nal(Tl) 3" $x$ 3" para amostra de pechblenda (Adams \& Gasparíni 1970).

Figure 2 - Gamma spectrum from ${ }^{238} \mathrm{U}$ natural decay series in radioactive equilibrium obtained with a 3" x 3" Nal(Tl) detector for a pitchblende sample (Adams \& Gasparini 1970).

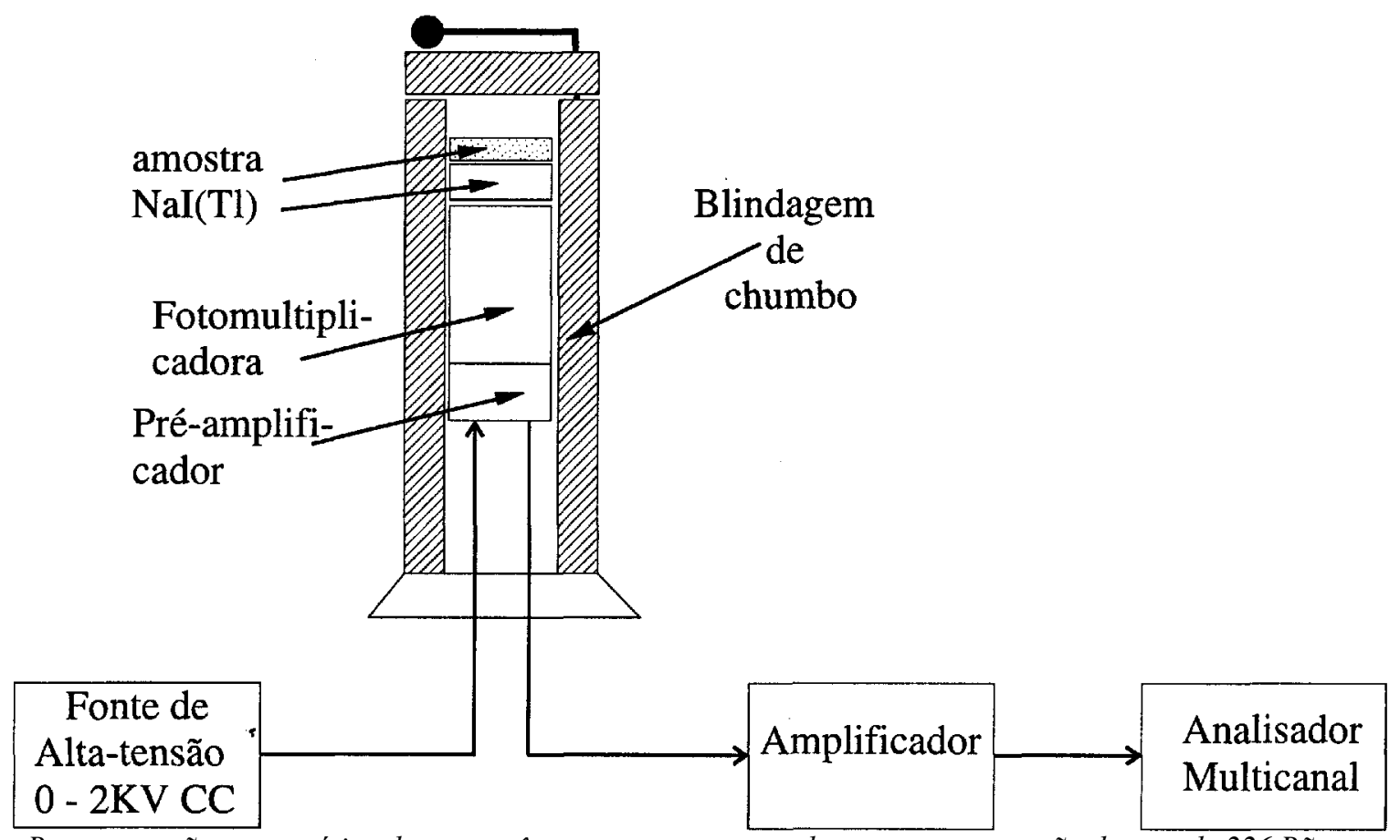

Figura 3 - Representação esquemática do espectrômetro gama empregado para a mensuração do teor de 226 Rã. Figure 3 - Sketch of the gamma spectrometer utilized to the evaluation of the ${ }^{226} \mathrm{Ra}$ content. 
Figura 4 - Curva de calibração do espectrômetro gama de

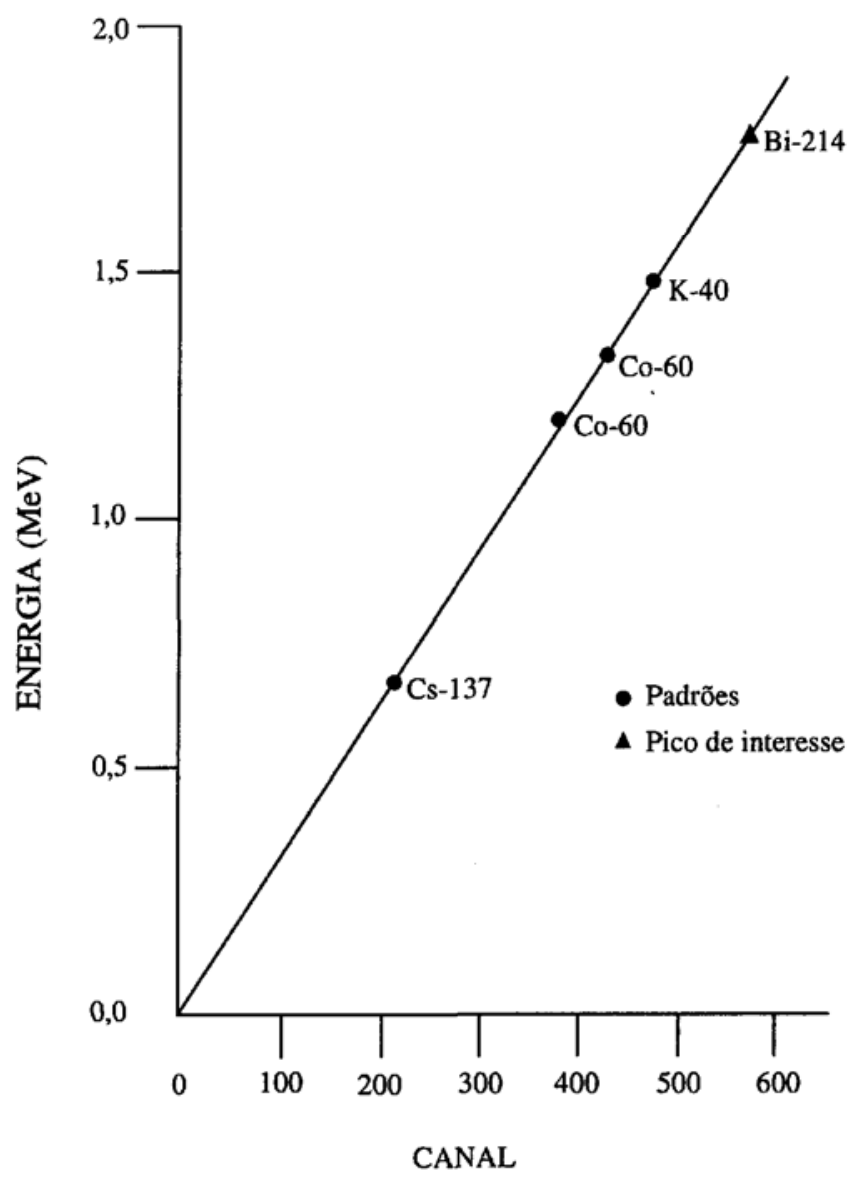

Figure 4 - Calibration curve of the gamma spectrometer in Energy vs. Channel.

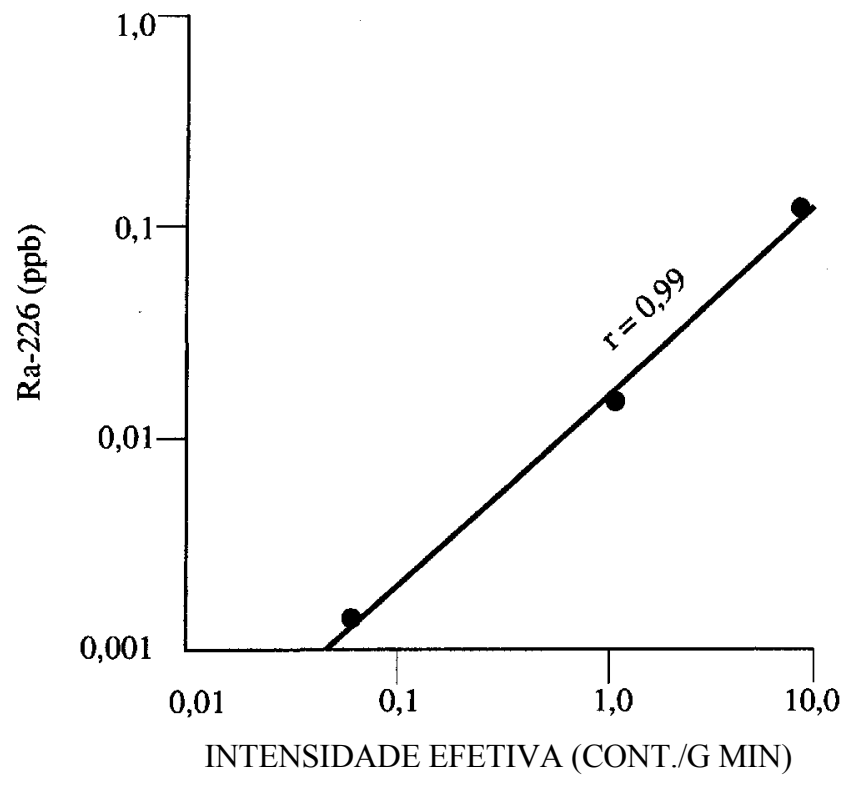

Figura 5 - Curva de calibração do teor de ${ }^{226}$ Ra em função da intensidade efetiva.

Figure 5 - Calibration curve of the ${ }^{226}$ Ra content vs. effective intensity.

valores estão representados em escala logarítmica, aos quais se ajustou por regressão linear a seguinte reta:

$$
\log \mathrm{C}=-1,76+0,91 \log (\mathrm{I})
$$

onde I é a intensidade efetiva em contagens/g.min e C é o teor de Ra-226 em ppb.

Resultados O teor de Ra-226 nas amostras foi calculado empregando-se a equação (5) e os valores da intensidade efetiva determinados a partir das contagens líquidas discriminadas na Tabela 6 . Conforme se nota, o valor mais elevado foi encontrado para a amostra de pseudo-leucita tinguaíto, que é próximo ao do padrão de menor concentração, isto é, 109-A. Se o equivalente urânio $(\mathrm{eU})$ fosse o parâmetro mensurado, então, valores entre 1 e 3,4 ppm seriam obtidos, os quais são comparáveis aos determinados por outros pesquisadores que trabalharam na área do planalto de Poços de Caldas. Por

Tabela 6 - Teor de ${ }^{226}$. Ra nas rochas do planalto de Poços de Caldas analisadas neste trabalho.

Table $6-{ }^{226} \mathrm{Ra}$ content in rocks from Poços de Caldas plateau analysed in this paper.

\begin{tabular}{l|c|c|c|c|c}
\hline \multicolumn{1}{c|}{ Amostra } & $\begin{array}{c}\text { Massa da amostra } \\
(\mathrm{g})\end{array}$ & $\begin{array}{c}\text { Tempo de } \\
\text { contagem } \\
(\mathbf{m i n})\end{array}$ & $\begin{array}{c}\text { Contagens líquidas } \\
(\mathrm{cpm})\end{array}$ & $\begin{array}{c}\text { Intensidade efetiva } \\
\left(\frac{\text { contagens }}{\mathrm{g} \cdot \mathrm{min}}\right)\end{array}$ & $\begin{array}{c}\text { Ra-226 } \\
\text { (ppb) }\end{array}$ \\
\hline Arenito silicificado & 84,74 & 166,7 & 2,95 & 0,035 & 0,000785 \\
Fonólito & 87,36 & 166,7 & 1,24 & 0,014 & 0,00034 \\
Nefelina sienito & 94,73 & 166,7 & 4,99 & 0,053 & 0,00114 \\
Pseudo-leucita & 97,04 & 166,7 & 5,28 & 0,054 & 0,00116 \\
tinguaíto & & & & & \\
\hline
\end{tabular}


exemplo, Schorscher \& Shea (1991) apresentaram resultados para o teor de urânio mensurados por fluorescência de raios $\mathrm{X}$ em 4 amostras de nefelina sienitos e 6 amostras de fonólitos procedentes da mesma área, tendo sido verificado que para apenas uma amostra o teor de urânio correspondeu a 12 ppm, enquanto que para as demais os teores situaram-se abaixo do limite de detecção (apesar de não especificado é possível que corresponda a 5 ppm).

DISCUSSÃO Emanação de ${ }^{222}$ RO Os mecanismos que podem ocasionar a emanação de Rn dos fragmentos de rocha e consequentemente contribuir para o seu teor nas águas são:

(1)Recuo do ${ }^{222} \mathrm{Rn}$ após o decaimento alfa dos átomos de

Rã próximos à superfície, isto é, posicionados à distância de cerca de 0,036 um correspondente à de recuo do ${ }^{222} \mathrm{Rn}$;

(2) Difusão do ${ }^{222} \mathrm{Rn}$ através do retículo cristalino, a partir de locais distanciados da superfície da rocha;

(3) Difusão do ${ }^{222} \mathrm{Rn}$ ao longo de defeitos cristalinos, contornos de grãos ou microfraturas distanciados da superfície da rocha.

$\mathrm{O}$ gradiente de concentração resultante da difusão de ${ }^{222} \mathrm{Rn}$ num meio isotrópico infinito pode ser avaliado pela equação:

$$
C_{x}=C_{0} e^{-x / L}
$$

onde $\mathrm{C}_{\mathrm{x}}$ e $\mathrm{C}_{0}$ são as concentrações de ${ }^{\llcorner\iota \iota} \mathrm{Rn}$ numa distância $\mathrm{x}$ e $\mathrm{O}$ servindo como referência e $\mathrm{L}$ corresponde à trajetória de difusão do Rn (em cm). Essa trajetória é definida como:

$$
\mathrm{L}=(\mathrm{D} / \lambda)^{1 / 2}
$$

onde D é o coeficiente de difusão do ${ }^{222} \mathrm{Rn}\left(\mathrm{cm}^{2} \mathrm{~s}^{-1}\right)$ e $\lambda$, é a constante de decaimento do ${ }^{222} \mathrm{Rn}\left(0,18\right.$ dias $\left.^{-1}\right)$. Portanto, a concentração de ${ }^{222} \mathrm{Rn}$ se reduz por um fator de 0,37 numa distância $\mathrm{x}=\mathrm{L}=1 \mathrm{~cm}$, podendo apenas $5 \%$ do ${ }^{222} \mathrm{Rn}$ atingir por processos difusivos uma distância $5 \mathrm{~L}$ a partir de sua origem. Valores típicos de $\mathrm{D}$ e $\mathrm{L}$ para $\mathrm{o} \mathrm{Rn}$ na água são, respectivamente, $10^{-5} \mathrm{~cm}^{2} \mathrm{~s}^{-1}$ e $2,18 \mathrm{~cm}$ (Tanner 1964).

O mecanismo de difusão no retículo não deve contribuir significativamente para a liberação de ${ }^{222} \mathrm{Rn}$ da rocha, pois, a constante de difusãpno retículo cristalino é extremamente pequena, isto é, $10^{-20} \mathrm{~cm}^{2} \mathrm{~s}^{-1}$ segundo Jost (1960), o que resulta num valor de $\mathrm{L}=7 \times 10^{-8} \mathrm{~cm}$, também pequeno e menor que a distância de recuo do ${ }^{222} \mathrm{Rn}$ em silicatos. Para amostras de calcário do Carbonífero estudadas por Zereshki (1983), apenas o processo de recuo alfa do Rn não é suficiente para justificar o teor de ${ }^{222} \mathrm{Rn}$ encontrado na água em contato com a rocha, porque um valor de $10^{-7} \mathrm{~cm}^{2} \mathrm{~s}^{-1}$ foi experimentalmente determinado para a constante de difusão do Rn nessa rocha, o qual possibilita obter $\mathrm{L}=0,22 \mathrm{~cm}$, resultados que são

$$
\lambda_{\mathrm{Ra}} \mathrm{N}_{\mathrm{Ra}}=\lambda \mathrm{N}
$$

superiores aos referidos para a difusão no retículo cristalino e atribuídos à difusão intergranular neste material.

Coeficientes de emanação de radônio Para se avaliar a geração de radônio em rochas e minerais e transferência para a água, uma importante premissa refere-se ao estabelecimento de equilíbrio radioativo dos radionuclídeos na série do urânio no sistema água-rocha, o que implica no fato de que a atividade do radônio na água deve se tornar igual à atividade do rádio na rocha matriz. Assim, é possível escrever:

onde $\lambda R_{a}$ e $X$ representam, respectivamente, a constante de decaimento radioativo do $\mathrm{Rã}$ e $\mathrm{Rn}$, e $\mathbf{N R}_{\mathbf{a}}$ e $\mathrm{N}_{226}$ representam, respectivamente, o número de átomos de ${ }^{226} \mathrm{Ra}$ e sentam, respectivamente, o número
${ }^{222} \mathrm{Rn}$ presentes no sistema água-rocha.
Contudo, a taxa de liberação de radônio para a fase líquida é menor que a sua taxa de produção na fase sólida (em virtude do decaimento de rádio) indicando que ocorre a retenção de radônio na fase sólida antes que se processe o seu escape para a água. $O$ coeficiente de emanação constitui um importante fator utilizado para expressar a fração de átomos de radônio formada no sólido e transferida para a água, o qual, segundo Wanty et al. (1992) pode ser expresso por:

$$
\mathbf{E}=\frac{\left({ }^{222} \mathbf{R n}\right)_{\text {água }}}{\left({ }^{222} \mathbf{R n}\right)_{\text {agua }}+\left({ }^{222} \mathbf{R n}\right)_{\text {rocha }}}
$$

Os resultados expressos na Tabela 5 para o ${ }^{222} \mathrm{Rn}$ liberado pelas rochas do planalto de Poços de Caldas sob condições controladas de laboratório podem ser utilizados para a determinação do coeficiente de emanação, desde que o teor de

$\mathrm{Rn}$ na rocha matriz também seja conhecido e expresso na mesma unidade. Esse parâmetro pode ser avaliado a partir dos resultados apresentados na Tabela 6 , tendo em vista que antes da realização das medidas espectrométricas gama aguardou-se o intervalo de tempo necessário para o estabelecimento do equilíbrio radioativo entre os nuclídeps ${ }^{226} \mathrm{Ra},{ }^{222} \mathrm{Rn}$ e Bi da série do urânio. Assim, o teor de Rã para cada amostra de rocha analisada pode ser convertido para atividade de

Rã, correspondendo os valores obtidos (entre 0,00034 e $0,00116 \mathrm{ppb}$ ) a $480 \mathrm{pCi}$ para o arenito silicificado, $187 \mathrm{pCi}$ para o fonólito, $773 \mathrm{pCi}$ para o nefelina sienito e $751 \mathrm{pCi}$ para o pseudo-leucita tinguaíto. Consequentemente, é possível estimar um coeficiente de emanação de 0,90 para o arenito silicificado e fonólito, de 0,69 para o nefelina sienito e de 0,53 para o pseudo-leucita tinguaíto.

Correlações e desenvolvimento futuro Os valores entre 0,53 e 0,90 para o coeficiente de emanação de radônio das rochas do planalto de Poços de Caldas são superiores àqueles determinados por Bonotto \& Andrews (1996) para amostras de calcário do Carbonífero, bem como à maioria daqueles referidos por Wanty et al. (1992) que consideraram que $\mathbf{E}$ é quase sempre menor que 0,5 , e usualmente menor que 0,3 .

Apesar do pequeno número de amostras analisadas, realizaram-se testes estatísticos envolvendo a determinação do coeficiente de correlação de Pearson $\mathbf{r}$ e a avaliação da hipótese nula de não correlação, a um nível de significância de 0,05 (Siegel 1956), visando apontar possíveis tendências de influência na atividade do ${ }^{222} \mathrm{Rn}$ liberado para a água dos seguintes parâmetros controlados nos experimentos realizados: massa total dos fragmentos utilizados em cada experimento de transferência de ${ }^{222} \mathrm{Rn}$, área superficial total de contato entre a rocha e a água, área superficial específica das rochas utilizadas nos experimentos de transferência e teor de

${ }^{226} \mathrm{Ra}$ nas rochas. Embora correlações não significativas foram obtidas entre estes parâmetros, quando os testes foram realizados com o coeficiente de emanação de radônio ao invés da atividade do $\mathrm{Rn}$ liberado para a água, verificaram-se tendências de correlação inversa com a massa total de rocha utilizada $(r=-0,71)$ e com o teor de ${ }^{226}$ Ra na rocha $(r=-0,83)$ bem como correlação direta com a área superficial específica das rochas $(r=0,75)$. Uma boa correspondência entre esse último parâmetro e a atividade do ${ }^{222} \mathrm{Rn}$ liberado de fragmentos de calcário do Carbonífero para a água foi encontrada por Bonotto \& Andrews (1996). Já as tendências de correlação inversa refletem sobretudo a natureza da rocha matriz, e, nesse sentido outros fatores não investigados certamente devem estar afetando a transferência de radônio para as águas, por exemplo, aqueles envolvendo propriedades microscópicas 
como rede de nanoporos ou distribuição não homogênea de ${ }^{226} \mathrm{Ra}$ nos sólidos. Enfatizando estes aspectos, Rama \& Moore (1984) propuseram que o radônio difunde para a atmosfera ou água por intermédio de uma rede de nanoporos nas rochas e minerais com 100 a 200 angstroms de largura, muitos dos quais interceptam a sua superfície originando uma grande área superficial interna no sólido (cerca de $10 \mathrm{~m}$ por $\mathrm{cm}$ ), muito maior que a área superficial externa calculada a partir da distribuição de tamanho das partículas. Porém, Krishnaswami \& Seidemann (1988) consideraram que a alta emanação de ${ }^{222} \mathrm{Rn}$ das rochas e minerais responsável pelo seu suprimento para o ar ou água está relacionada com o enriquecimento de ${ }^{226} \mathrm{Ra}$ nos contornos dos grãos ou microfraturas ao invés de uma extensa rede de nanoporos. Além disso, os estudos conduzidos por Pereira (1977) em amostras de rocha do Campo do Cercado (Mina Osamu Utsumi) no planalto de Poços de Caldas, envolvendo a distribuição de elementos radioativos pela técnica de autorradiografia, mostraram que uma distribuição pontual e intensa não propiciou a obtenção de coeficiente de emanação elevado, enquanto que uma distribuição uniforme permitiu determinar maiores coeficientes de emanação, reforçando, então, a necessidade de realização de investigações sistemáticas enfocando também a importância das propriedades microscópicas dos sólidos para a liberação de radônio para a água, além dos parâmetros considerados neste trabalho. Um aspecto adicional motivando a realização dessas pesquisas constitui os elevados coeficientes de emanação correspondentes a 0,90 obtidos para duas amostras, os quais necessitam ser esclarecidos pois valores dessa ordem de grandeza são pouco referidos em literatura. Finalmente, tentativas de se estabelecer analogias entre o comportamento do radônio e dos isótopos de argônio nos sólidos constituiriam tópicos de interesse para a geocronologia.

CONCLUSÃO As metodologias utilizadas no presente trabalho denotaram ser apropriadas para a determinação dos teores de ${ }^{226} \mathrm{Ra}$ (mediante a técnica de espectrometria gama)

e do coeficiente de emanação de radônio das seguintes rochas do planalto de Poços de Caldas: arenito silicificado, fonólito, nefelina sienito e pseudo-leucita tinguaíto. As experiências de transferência de radônio destas rochas foram conduzidas sob as seguintes condições controladas em laboratório: massa total de rocha em contato com água, área superficial total de rocha em contato com a água e tempo para o ${ }^{222} \mathrm{Rn}$ atingir o equilíbrio radioativo com o ${ }^{226} \mathrm{Ra}$. Os valores obtidos situaram-se entre 849 e 4117 pCi para a atividade do ${ }^{222} \mathrm{Rn}$ liberado para a água, os ouais permitiram determinar coeficientes de emanação de ${ }^{222} \mathrm{Rn}$ entre 0,53 e 0,90 , superiores à maioria daqueles referidos em literatura. As correlações efetuadas entre esse parâmetro e a massa total de rocha em contato com a água, a área superficial total de rocha em contato com a água, a área superficial específica e o teor de Rã nas rochas indicaram algumas tendências de relação direta e inversa, reforçando observações de investigações prévias realizadas e sugerindo a necessidade de trabalhos futuros envolvendo outros parâmetros não considerados nos experimentos realizados.

Agradecimentos Os autores expressam seus sinceros agradecimentos ao Prof. Dr. Wolfgang C. Pfeifer pela cessão dos padrões de ${ }^{226} \mathrm{Ra}$ utilizados na calibração do sistema e ao

Prof. Dr. Antonio Misson Godoy pelo auxílio na coleta de amostras.

\section{REFERÊNCIAS}

Adams, J.A.S. \& Gasparini, P. 1970. Gamma-Ray Spectwmetry of Rocks. Amsterdam, Elsevier Publishing Company. 279 p.

Andrews, J.N. \& Wood, D.F. 1972. Mechanism of radon release in rock matrices and entry into ground waters. Inst. Min. Metall. Trans., B 81: 198.

Bonotto, D.M. \& Andrews, J.N. 1993. The mechanism of ${ }^{234} \mathrm{U} /{ }^{238} \mathrm{U}$ activity ratio enhancement in karstic limestone groundwater. Chem. Geol. (hot. Geosci. Sec.), 103: 193-206.

Bonotto. D.M. \& Andrews, J.N. 1996. The implications of the laboratory ${ }^{22} \mathrm{Rn}$ flux measurements to the radioactivity in groundwaters: the case of a karstic limestone aquifer. Appl. Geochem., in press.

Bruno, R.C. 1983. Sources of indoor radon in houses: a review. / AirPollitt. Control Assoc., 33: 105-109.

Bushee, J. 1971. A Geochronological Study of the Alkaline Massif of Poços de Caldas, Brazil. Berkeley. 145 p. (Ph.D. Thesis, University of California).

Cruz, W.B. \& Peixoto, C.A.M. 1989. As águas termais de Poços de Caldas, MG estudo experimental das interações água-rocha. Rev. Bras. Geoc., 19: $76-86$.

Dalrymple, G.B. \& Lanphere, M.A. 1969. Potassium-Argon Dating-Principles, Techniques and Applications to Geochronology. San Francisco, W.H. Freemann and Company. 258 p.

Durrance, E.M. 1986. Radioactivity in Geology -Principles and Applications. New York, Wiley. 441p.

Ellert, R. 1959. Contribuição à geologia do maciço alcalino de Poços de Caldas. São Paulo, FFCLH/USP. 64 p. (Boi. 237).

Ferreira, F.J.F. 1991 . Aerogamaespectrometria e Aeromagnetometria de um Trato Ocidental do Pré-Cambriano Paulista. São Paulo. 150 p. (Tese de Doutoramento, Universidade de São Paulo).

Gesell, T.F. \& Prichard, H.M. 1980. The contribution of radon in tap water to indoor radon concentrations. In: SYMPOSIUM ON THE NATURAL RADIATION ENVIRONMENT, HI. Houston, 1978. Proceedings... USDOE. p. 5-56.
Hahn, O. \& Miiller, H.O. 1923. Eine neue methode zum studium der oberflãche und oberflàchenãnderung feinverteilter niederschlâge. $Z$. Elektrochem.,29: 189-192.

Hiodo,F.Y. 1989. Desenvolvimento de Instrumentação em Geofisica Nuclear e sua Aplicação no Morro do Ferro. São Paulo. 177p. (Tese de Doutoramento, Universidade de São Paulo).

Holmes, D.C.; Pitty, A.E. \& Noy, D.J. 1991. Geomorphological and tiydrogeological Features of the Poços de Caldas and the Osamu Utsumi Mine and Morro do Ferro Analogue Study Sites, Brazil. Uppsala, 8KB - Swedish Nuclear Fuel and Waste Management Co. 55 p. (Poços de Caldas Project, Series Rep. 5).

Jost, W. 1960. Diffusion in solids, liquids, gases. New York, Academic Press. Krishnaswami, S. \& Seidemann, D.E. 1988. Comparative study of ${ }^{222} \mathrm{Rn}$, ${ }^{40} \mathrm{Ar},{ }^{39} \mathrm{Ar}$ and ${ }^{37} \mathrm{Ar}$ leakage from rocks and minerals: implications for the role of nanopores in gas transport through natural silicates. Geochim. Cosmochim. Acta, 52: 655-658.

Lima, J.L.N. \& Bonotto, D.M. 1995. Etapas Analíticas para Mensuração de Rn-222 e Uso na Avaliação da Radioatividade das Águas de Águas da Prata (SP). Geochim. Brasil, no prelo.

Nazaroff, W.W. \& Nero, A.V. Jr. 1988. Radon and its decay products in indoor air. New York, John Wiley \& Sons. 518 p.

Nazaroff, W.W.; Doyle, S.M.; Nero, A.V. Jr.; Sexto, R.G. 1988. Radon entry via potable water. In: NAZAROFF, W.W. \& NERO Jr., A.V. eds. Radon and its decay products in indoor air. New York, John Wiley \& Sons.

Oliveira, A.G. 1974. Mineralização de urânio e molibdênio no planalto de Poços de Caldas-MG. In: CONGR.BRAS.GEOL., 28 Porto Alegre, 1974. Anais... Porto Alegre, SBG. v.1, p. 207-221.

Partridge, I.E.; Horton, T.R. \& Sensintaffer, E.L. 1979. A study ofradon-222 released from water during typical household activities. U.S. Environmental Protection Agency. (Tech. Note ORP/EERF-79-1).

Pascholati, E.M. 1990. Caracterização Geofisica da Suite Intrusiva de Itu. São Paulo. 135 p. (Tese de Doutoramento, Universidade de São Paulo). 
Pereira, E.B. 1977. Caracterização da produção do Rn-222 no Campo do Cercado C-09, Poços de Caldas, MG. São Paulo. 120 p. (Tese de Mestrado, Universidade de São Paulo).

Prichard, H.M. 1987. The transfer of radon from domestic water to indoor air. /.Am. Water Works Assoc., 79: 159-161.

Rama \& Moore, W.S. 1984. Mechanism of transport of U-Th series radioisotopes from solids into groundwater. Geochim. Cosmochim. Acta, 48: 395-399.

Schorscher, H.D. \&Shea, M.E. 1991. The regional geology, mineralogy and geochemistry of the Poços de Caldas alkaline caldera complex, Minos Gerais, Brazil. Uppsala, SKB-Swedish Nuclear Fuel and Waste Management Co. 36 p. (Poços de Caldas Project, Series Rep. 1).

Siegel, S. 1956. Nonparametric statistics for the behavioral sciences. New York, McGrawHill Book Company. 312p.

Tanner, A.B. 1964. Radon migration the ground: a review. In: ADAMS, J.A.S. \& LOWDER, W.M. eds. The Natural Radiation Environment. Chicago, Chicago University Press.
Tanner, A.B. 1980. Radon migration in the ground: a supplementary review. In: SYMPOSIUM ON THE NATURAL RADIATION ENVIRONMENT, III. Houston, 1978. Proceedings... USDOE.

Wahl, A.C. \& Bonner, N.A. 1951. Radioactivity Applied to Chemistry. New York, John Wiley \& Sons. p. 284-319.

Wanty, R.B.; Lawrence, E.P. \& Gundersen, L.C.S. 1992. A theoretical model for the flux of radon from rock to groundwater. In: Gates, A.E. \& Gundersen, L.C.S. eds. Geologic controls on radon. Boulder.

Zereshki, A. 1983. The solution of ${ }^{22} R n$ by groundwaters. Bath. (Ph. D. thesis, University of Bath).

Manuscrito A914

Recebido em 22 de abril de 1997

Revisão do autor em 10 de julho de 1997

Revisão aceita em 12 de julho de 1997 